\title{
Cellular strain amplifies LPS-induced stress signaling in immature enterocytes: potential implications for preterm infant NCPAP
}

\author{
Alicia M. Alcamo' ${ }^{1}$ Brandon L. Schanbacher ${ }^{2}$, Hong Huang², Craig A. Nankervis² ${ }^{2}$ John A. Bauer ${ }^{2}$ and Peter J. Giannone ${ }^{2}$
}

\begin{abstract}
BACKGROUND: Recent clinical observations of increased necrotizing enterocolitis (NEC) incidence in some nasal continuous positive airway pressure (NCPAP) patients raise concerns about whether the related abdominal distension is benign or contributes to NEC. We tested the hypothesis that mechanical strain causes an exaggerated enterocyte inflammatory response and decreased enterocyte growth and proliferation in the absence and presence of lipopolysaccharide (LPS).
\end{abstract}

METHODS: First we used a confluent enterocyte (IEC-6) monolayer to investigate effects of strain on inflammatory cytokine production and Toll-like receptor 4 (TLR-4) gene expression. Then we used a low seeding density to measure cell growth and proliferation. Ten percent mechanical strain was applied.

RESULTS: Significant increases in interleukin (IL)-8 and in IL-6 were observed after 8 and $24 \mathrm{~h}$ of cellular strain, respectively, and maintained throughout the study. TLR-4 expression was increased at $48 \mathrm{~h}$. Mechanical strain led to slower proliferation and division whereas LPS alone had minimal effects. The responses of LPS and strain were supra-additive, suggesting synergistic cellular effects.

CONCLUSION: We speculate intestinal distension associated with the use of NCPAP, especially in the presence of abnormal gut colonization, may result in increased inflammatory cytokine production and be a contributing factor to neonatal intestinal morbidities.

nfants born prematurely often require some form of respira-
tory support in the first weeks of life, and one such approach is
the use of nasal continuous positive airway pressure (NCPAP).
This system improves gas exchange through dilation of the
respiratory tree (via increased pressure through the nasal
pharynx) and prevention of expiratory lung collapse, thereby
decreasing respiratory effort and improving oxygenation. Its
use in premature infants is important because of the difficulty
these newborns have with maintaining upper airway patency
and functional residual capacity (1). As compared with the use
of mechanical ventilators, NCPAP facilitates early extubation
and has decreased incidence of acute lung injury and related
complications (2-5). Observational studies have also shown that the use of NCPAP in place of mechanical ventilation has resulted in a reduction of oxygen dependence for preterm infants at $28 \mathrm{~d}(2,4)$. Collectively, these findings have promoted a transition to the use of NCPAP within most neonatal intensive care settings wherever feasible.

Although NCPAP has definite benefits with respect to pulmonary and oxygenation status in preterm infants, its use is also associated with abdominal distension, commonly referred to as "CPAP belly syndrome." This abdominal distension has been observed to occur $\sim 4-7 \mathrm{~d}$ following initiation of NCPAP and involves dilation of both the small and large bowel (6). It has been suggested that CPAP belly syndrome is related to the functional immaturity and poor motility of the intestine and trapping of air driven by positive pressure at the nasal cavity. The condition appears to be most severe in the smallest and most premature infants, as Jaile et al. (6) noted that abdominal distension occurred within $83 \%$ of infants weighing $<1,000 \mathrm{~g}$, but only in $14 \%$ of infants weighing $>1,000 \mathrm{~g}$. In this patient population, NCPAP can cause gastrointestinal air-trapping and distended abdominal circumference by as much as $30 \%$ (P.J. Giannone, B.L. Schanbacher, J.A. Bauer et al., unpublished data). Given the rapidly expanded use of NCPAP in neonatal nurseries, and the increasing prevalence of preterm births worldwide, a closer study of this gastrointestinal challenge in the youngest of patients appears warranted, and thus far the findings are controversial. For example, Jaile et al. (6), found no increase in gastrointestinal issues for those infants who developed abdominal distension on NCPAP, suggesting that CPAP belly syndrome is a benign entity. In contrast, Aly et al. (3) found an association of NCPAP use and increased incidence of necrotizing enterocolitis (NEC), the most common gastrointestinal disease that afflicts newborn infants. Approximately $5-10 \%$ of all infants with a birth weight $\leq 1,500 \mathrm{~g}$ are diagnosed with NEC, and this is associated with a mortality rate of $15-30 \%(7,8)$. The mechanisms of NEC are not clearly defined, but specific risk factors include prematurity, ischemia, aggressive enteral feedings, and infection; inflammation-driven tissue injury and necrosis are predominant mechanisms (9-11). The vulnerability of premature infants to the development of 
NEC has been attributed to an immature gastrointestinal tract vulnerable to potential overgrowth of pathogens, especially as a result of poor motility $(10,12)$.

Recent clinical observations of increased NEC incidence in some NCPAP patients raise concerns about whether the related abdominal distension is benign in all infants, or if it contributes to NEC pathogenesis. This may be of special concern in the extremely premature, wherein abdominal wall tone and intestinal maturation are both minimal and organ distension may be most extensive. To our knowledge, no observational or randomized trial study has been conducted to specifically address potential differences in the occurrence or progression of NEC with the use of NCPAP. Furthermore, the potential interactions of intra-luminal intestinal pressure and other stimuli that are suspected contributors to intestinal injury and risk factors for NEC have not been investigated. For these reasons we studied the effects of mechanical strain on isolated immature intestinal epithelial cells in vitro. Our objective herein was to test the hypothesis that mechanical strain causes an exaggerated enterocyte inflammatory response, in the absence and presence of inflammatory stimulation via lipopolysaccharide (LPS).

\section{RESULTS}

Initial studies were conducted to determine the optimal starting conditions for the neonatal rat enterocytes to adequately adhere before applying strain. We found that seeding with at least 250,000 cells $/ \mathrm{cm}^{2}$ of membrane surface, and allowing a 48-h post-seeding time before applying strain reliably provided fully confluent cell monolayers. Preliminary investigations also showed that the monolayer was maintained throughout the cyclic strain conditions we employed (20\% distension, $3 \mathrm{~s} /$ cycle, 10 cycles/min) with at least $90 \%$ confluence throughout the study period.

Time-dependent effects of mechanical strain on production of inflammatory cytokines by already confluent neonatal rat intestinal epithelial cells are shown in Figure 1. Statistically significant increases in interleukin (IL)-6 (Figure 1a) and in IL-8 (Figure 1b) were observed after 24 and $8 \mathrm{~h}$ of cellular strain, respectively, and this was maintained throughout the remainder of the $48 \mathrm{~h}$ studied. The increases in IL- 8 were found to be more extensive than those in IL-6, with nearly 10-fold higher levels of IL-8 from this cell type in response to mechanical strain. Under the conditions employed the total number of adherent cells was not different from that of the control treatment (e.g., cells cultured on identical membranes but with no strain forces applied, see (Figure 1c)). Significantly increased gene expression of Toll-like receptor $4(T L R-4)$ was also observed in strained enterocytes after $48 \mathrm{~h}$ as compared with static control conditions (Figure 1d).

Concentration- and time-dependent changes of IL- 6 and IL-8 during LPS exposure to rat neonatal enterocytes under static conditions (e.g., in the absence of strain) are shown in Figure 2. Statistically significant increases in both cytokines were observed by $24 \mathrm{~h}$ of treatment and were maintained until $48 \mathrm{~h}$. In relative comparison, the LPS-concentration response
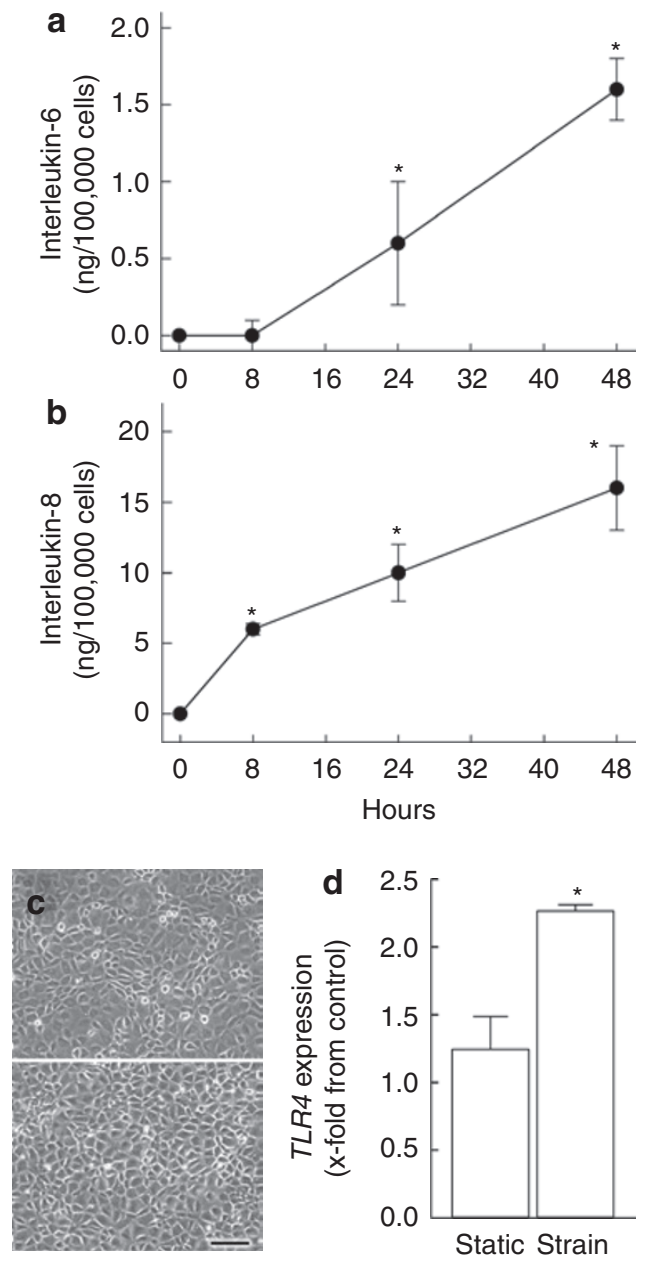

Figure 1. Effects of cellular strain alone on rat neonatal enterocytes. (a) Time-dependent increases in interleukin (IL)-6. (b) Time-dependent increases in IL-8. (c) Representative images of static (upper panel) vs. strained enterocyte monolayer following $48 \mathrm{~h}$. Scale bar $=100 \mu \mathrm{m}$. (d) Increased Toll-like receptor 4 gene expression following $48 \mathrm{~h}$ of strain vs. static conditions. All panels, $n=6-12$, mean $\pm \mathrm{SE} ;{ }^{*} P<0.05$ as compared with time 0 static control.

was more robust with respect to IL-8 expression than IL-6 expression under these conditions.

Synergistic effects of cellular strain and LPS exposures with respect to cytokine expression by confluent rat neonatal enterocytes are shown in Figure 3. Confluent enterocyte monolayers were exposed to cyclic strain, LPS, or both for 24 and $48 \mathrm{~h}$. LPS caused time-dependent increases in IL- 6 and IL-8 production at 10 and $150 \mu \mathrm{g} / \mathrm{ml}$; these LPS responses were substantially increased when combined with the presence of cellular strain. In most cases (with the exception of $10 \mu \mathrm{g} / \mathrm{ml}$ LPS and IL-6 response) the response to the combination of LPS and strain was supra-additive as compared with the two stimuli used alone; suggesting that this combined challenge caused synergistic cellular effects.

The effects of cellular strain, LPS, or their combination on enterocyte growth and proliferation before confluence are shown in Figure 4. At fixed time points, numbers of cells per field of view were recorded (Figure 4a), and actively dividing 

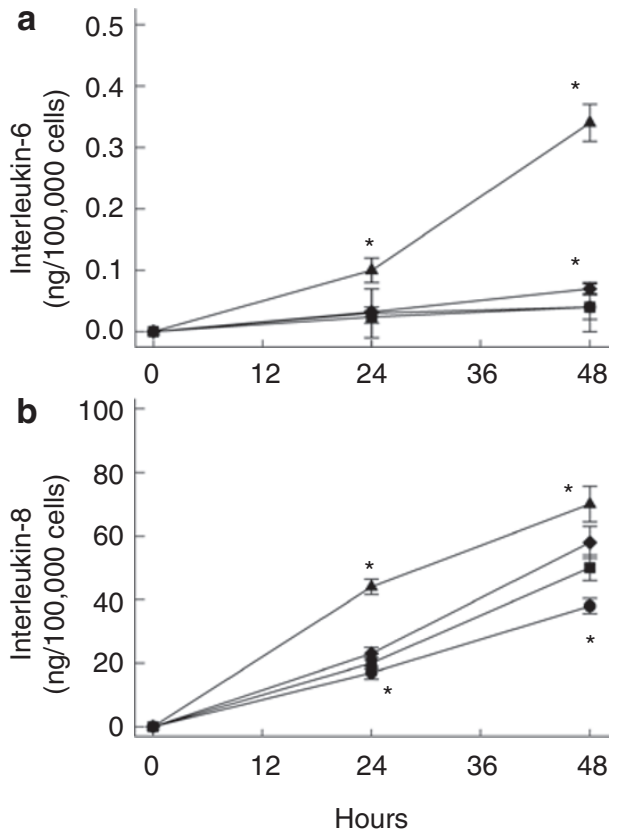

Figure 2. Effects of lipopolysaccharide (LPS) alone on rat neonatal enterocytes. Time-dependent increases in (a) interleukin (IL)-6 or (b) IL-8 during various LPS exposures ( $n=6-12$, mean \pm SE) (filled circle $=10 \mu \mathrm{g} / \mathrm{ml}$, filled square $=50 \mu \mathrm{g} / \mathrm{ml}$, filled diamond $=100 \mu \mathrm{g} / \mathrm{ml}$, filled triangle $=150$ $\mu \mathrm{g} / \mathrm{ml})$. All panels, ${ }^{*} P<0.05$ as compared with time 0 static control.
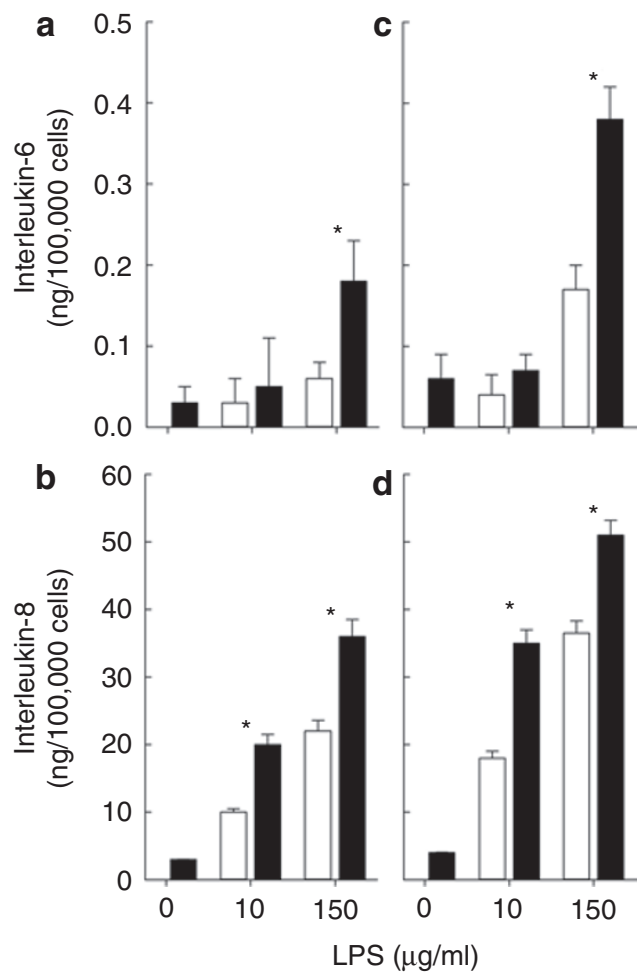

Figure 3. Effects of combined treatment of lipopolysaccharide (LPS) and cellular strain on rat neonatal enterocyte interleukin (IL)-6 and IL-8. Confluent monolayers were incubated with LPS for (a and $\mathbf{b}) 24 \mathrm{~h}$ or (c and d) $48 \mathrm{~h}$ in the absence (open bars) or presence (black bars) of cyclic strain. Supra-additive effects of the combined treatment were observed $\left(n=6-12\right.$, mean \pm SE). All panels, ${ }^{*} P<0.05$ as compared with the time-respective static control.
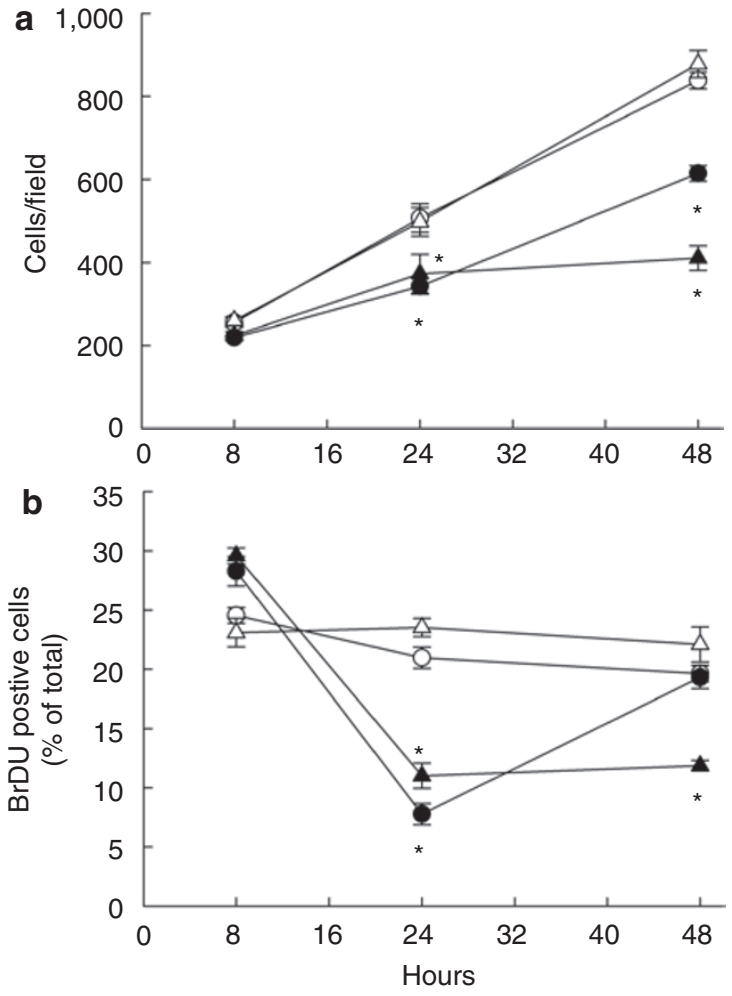

Figure 4. Effects of cellular strain or lipopolysaccharide (LPS) on subconfluent cell growth and proliferation in rat neonatal enterocytes. Cells were seeded at low seeding density; (a) numbers of cells/ $\times 100$ field of view and (b) 5'-bromo-2-deoxyuridine (BrDU)-positive cells were counted $(n=6-12$, mean \pm SD) (open circle $=$ nonstrained, $0 \mathrm{mg} / \mathrm{ml} \mathrm{LPS}$, open triangle $=$ nonstrained, $10 \mathrm{mg} / \mathrm{ml} \mathrm{LPS}$, filled circle $=$ strained, $0 \mathrm{mg} /$ $\mathrm{ml}$ LPS, filled triangle $=$ strained, $10 \mathrm{mg} / \mathrm{ml}$ LPS). All panels, ${ }^{*} P<0.05$ as compared with the respective static control.

cells were identified by $5^{\prime}$-Bromo-2-deoxyuridine (BrDU) incorporation (see Methods for details). Cellular strain alone, or in combination with LPS $10 \mu \mathrm{g} / \mathrm{ml}$, caused slower cell growth, with nearly half as many cells as the control by the 48-h time point (Figure 4a). Similarly, the percentage of BrDU positive cells in the cyclic strain treatment groups was approximately one-half of the control or LPS alone at $24 \mathrm{~h}$. Collectively, these data demonstrate that in neonatal enterocytes mechanical strain leads to slower cell growth and division whereas LPS alone has minimal effects under these conditions.

\section{DISCUSSION}

Over the past several decades, improvements in medical care have substantially increased survival and improved outcomes in preterm infants $(13,14)$. Unfortunately the incidence of necrotizing enterocolitis has actually risen slightly over this time period, most likely because of the fact that more premature infants are surviving the initial newborn period (15-17). The advent of NCPAP is one advance that has contributed to improved survival and neonatal outcomes as it can reduce the need or duration of mechanical ventilation, and may lessen the incidence of bronchopulmonary dysplasia $(2,3)$. However, the phenomenon of CPAP belly syndrome, likely due to passive 
air-trapping via the esophagus to the stomach and intestine, can cause abdominal distension of a preterm infant, postulated to be the result of minimal sphincter control and abdominal wall muscle mass and tone (6). Some epidemiological studies have concluded that this is a benign manifestation, but other observational studies have noted a potential increase in the rate of necrotizing enterocolitis in premature infants $(3,18-$ 22). Although leaders in the area of necrotizing enterocolitis have recently suggested that the pathophysiology involved is multifactorial, there may be subgroups of infants at higher risk of developing NEC due to settings that may allow for a more robust response of the innate immune system of the premature intestine in response to stress (23). To our knowledge, there have been no definitive trials to investigate the impact of NCPAP on the incidence or progression of NEC or any related gastrointestinal ailments in preterm infants. The goal of this study was to test the hypothesis that strain causes cellular stress responses that are already implicated in clinical NEC pathogenesis, either alone or in combination with an additional known stressor, LPS.

These isolated cell studies have shown that the mechanical strain caused by cyclic rhythmic deformation, in addition to exposure to a LPS stimulus causes alterations in the inflammatory cytokine profiles. We chose to study these immature rat intestinal epithelial cells (IEC-6) particularly as they originate from the crypt and this is the area of epithelial intestinal mucosa that would see the most strain during distension as opposed to cells within the villi. In addition, mucosal epithelial cells appear to play a significant role in newborn intestinal diseases associated with local inflammation $(23,24)$. We have demonstrated increased production of both rat IL- 6 and growth related oncogene/cytokine induced neutrophil chemoattractant 1 (human IL-8 equivalent) cytokines by the rat intestinal cell line in response to both strain and LPS alone. These cytokines were chosen because they have been shown to be elevated in response to LPS in the cell line by other investigators and have been elevated at $10-20 \%$ mechanical strain in other cell types in a dose-response fashion (25-27). Of note, mechanical strain in the absence of an LPS insult caused a significant increase in the production of the pro-inflammatory IL-8 cytokine, likely stimulated by pathways other than through TLR-4. More important, cytokine production in these immature intestinal epithelial cells occurs in an apparent synergistic manner when LPS is added to these cells as opposed to either strain or LPS alone. In addition, many studies have shown that TLR-4 expression is increased in the neonatal intestine with exposure to an LPS stimulus $(28,29)$. Furthermore, other studies have shown that there is increased TLR-4 expression in necrotizing enterocolitis, suggesting that it may be involved in the pathogenesis of the disease (30). Therefore, as we have demonstrated that mechanical strain causes a significant increase in the expression of TLR-4, we speculate this relates to the potential interaction between the inflammatory cytokine pathway and mechanical strain and may contribute to morbidities of the immature intestine.

Another important finding of our study was that mechanical strain significantly decreased immature intestinal cell growth in vitro. Similar to our findings involving cytokine expression, cellular strain was associated with decreased immature enterocyte proliferation. No effect on cell proliferation was seen in the presence of LPS alone. These findings again speak to the concept that the pathogenesis of intestinal problems is multifactorial in nature and may require multiple "hits" to progress to true injury and disease. This finding could significantly impact maturation of the intestinal barrier as well as play a detrimental role in wound healing in repair. It is also interesting to note that presence of cellular strain caused immature enterocytes to shift toward production of inflammatory cytokines, and away from growth and proliferation, seemingly as a "host-defense" response to this mechanical stress. Both of these responses could be considered detrimental to premature infant intestinal maturation. These two responses were not identical to the responses observed with LPS alone (which increased cytokine expression but had no effects on cell growth), and in fact these two stimuli were found in some aspects to be supra-additive. In addition, we observed that strain alone was sufficient to cause an increase in TLR- 4 expression by $48 \mathrm{~h}$ (Figure 1). Further studies to define the signaling pathways involved in strain-augmented LPS responses, and their contributions to NEC incidence and/ or progression are warranted.

It is interesting to note that the temporal profiles observed for the measured cytokines, TLR- 4 expression, and cell replication were not identical under the conditions we employed. These disparate profiles suggest that the signaling pathways involved are likely not identical for each of these analytes, and this is consistent with studies using other cell types or conditions that investigate stretch wherein both the nuclear factor kappa $\beta$ and mitogen activated protein kinases pathways have been implicated. In our experiments it is possible that the earlier upregulation of cytokine release served as an autocrine contributor to later increases in TLR-4 and/or modulator of cell growth and proliferation. Further studies to address these important mechanisms, and particularly to determine signaling interactions of LPS-mediated vs. stretch-mediated effects in this system are clearly warranted. Additional investigations using in vivo conditions and intact tissues to address this important clinical problem are also under way.

The pathophysiology of necrotizing enterocolitis is not well understood, which makes the prevention and treatment of this devastating disease very difficult. Because of this uncertainty, it is important to understand the effect these various exposures have on the intestinal epithelium. We postulate that the increased inflammatory cytokines released from the intestinal epithelium in response to mechanical strain, such as that caused by the use of NCPAP, may relate to the pathophysiology of intestinal diseases of the neonate, such as poor motility leading to feeding intolerance and even perhaps necrotizing enterocolitis. The fact that mechanical strain induces the expression of inflammatory cytokines and increases the expression of TLR-4 receptors allows us to speculate that intestinal distension associated with the use of NCPAP, especially in the presence of abnormal gut colonization, results in increased 
inflammatory cytokine production and may be a contributing factor to neonatal intestinal morbidities, such as necrotizing enterocolitis and feeding intolerance. It is known that the intestinal epithelium is under continuous and rapid turnover, and in infants (especially preterm infants) it is likely that cellular proliferation is a key component of maintained integrity and rapid maturation. Our data support the concept that intestinal stretch could delay this process, and potentially enhance the risk of more severe conditions such as NEC by predisposing the intestinal tissues to a subsequent challenge (such as LPS). This phenomenon is supported by the general working theory that NEC itself is not a disease of one specific origin but may be an overlay of multiple intestinal insults to a vulnerable patient. Further studies should be conducted and are currently under way to elucidate the relationship between the use of NCPAP and the possible role this modality may play regarding intestinal morbidities. Understanding the interactions between the pathways regulated by these stressors will be imperative for improving the treatment and management of this vulnerable population.

\section{METHODS}

\section{Isolated Cell Culture}

Immature rat intestinal epithelial cells were obtained from American Type Culture Collection (IEC-6, ATCC, Rockville, MD) and were maintained in complete Dulbecco's Modified Eagle's Medium with $10 \%$ fetal bovine serum, L-alanyl-L-glutamine, insulin, and penicillin/streptomycin in a humidified atmosphere of $5 \% \mathrm{CO}_{2}$ and $95 \%$ air at $37^{\circ} \mathrm{C}$. All studies were conducted using cells at less than passage 20.

To investigate the impact of cellular strain on enterocyte responses, we used two experimental conditions. The first was using a confluent monolayer, to investigate the effects of strain on inflammatory cytokine production and TLR-4 gene expression. The second was using a low seeding density to test the hypothesis that strain affects enterocyte growth and proliferation. When evaluating response to inflammatory stimuli, LPS was added at varying concentrations ranging from $10-150 \mu \mathrm{g} / \mathrm{ml}$.

\section{Application of Mechanical Strain}

Cells were seeded at a density of either 30,000 or 250,000 cells $/ \mathrm{cm}^{2}$ on type IV collagen-coated Bioflex II 6-well plates (Flexcell International, Hillsborough, NC). Preliminary experiments were conducted to determine conditions providing reliable and stable confluence throughout the study protocols employed. Before application of strain, cells were maintained for $48 \mathrm{~h}$ in order to allow them to reach confluence, which was confirmed by light microscopy before use. A $10 \%$ mechanical strain was applied using a Flexcell II System (Flexcell International) at a rate of 10 cycles $/ \mathrm{min}$. Each cycle consisted of $3 \mathrm{~s}$ of membrane deformation followed by $3 \mathrm{~s}$ with the membrane in a neutral state. In our preliminary studies, we investigated the relationship between degree of stretch conditions and cellular viability and maintenance of a stable monolayer in vitro. We found that the conditions we used herein provided reliable and reproducible cell numbers throughout the study.

\section{Cytokine Analysis}

At each time point with and without an LPS stress, cell culture media was collected for cytokine assays by enzyme-linked immunosorbent assay assays using manufacturer protocols. Commercial enzymelinked immunosorbent assay kits were used to determine rat IL-6 (BD, Franklin Lakes, NJ) and GRO/CINC-1, the rat ortholog to human IL-8 (R\&D Systems, Minneapolis, MN). Each well was analyzed separately and then the average from the wells in each treatment group was obtained. In all cases $n=6-9$ and intra- and interassay variations were $<7 \%$. Cytokine concentrations in cell-free media content were normalized to numbers of adherent cells at each time point (determined by nuclear cell counts using digital image analysis at the time of media collection).

\section{Detection of TLR-4 Gene Expression}

RNA was collected using Qiagen RNeasy Mini Kit (Qiagen, Valencia, CA) per kit instructions. Briefly, cells were lysed directly in wells (after removing media) and lysate was analyzed in triplicate. A total

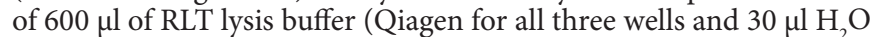
was used for elution of RNA for each sample. RNA quality was evaluated on a $1 \%$ agarose gel denatured with formaldehyde to ensure that $28 \mathrm{~s}$ and $18 \mathrm{~s}$ bands were present. Reverse transcriptase real-time PCR was used to detect the presence of TLR- 4 in the samples and was completed using the MuLV Reverse Transcriptase (Applied Biosystems, Carlsbad, CA). Using one-step PCR SYBR green on the iCycler (BioRad Laboratories, Hercules, CA), real-time PCR was performed. The steps included a 3-min denaturation at $95^{\circ} \mathrm{C}$ step, followed by a 40-cycle thermal cycling that was composed of a 30-s denaturation at $95^{\circ} \mathrm{C}, 10$-s annealing at $61^{\circ} \mathrm{C}$, and a 30 -s extension at $72{ }^{\circ} \mathrm{C}$. The primer used for the TLR-4 gene was a 160-basepair product with the following sequences: up 5' GGA TTT ATC CAG GTG TGA AA 3' and down 5' TTT GTC TCC ACA GCC ACC A 3' (Integrated DNA Technologies, Coralville, IA). A housekeeping ribosomal protein L30 gene (RPL30) was used for normalization of results. This was a 189basepair product with the following sequences: up 5' GAT CAG ACA AGG CAA AGC GA $3^{\prime}$ and down 5' TCA ATG ATA GCC AGT GTG CA 3' (Integrated DNA Technologies).

\section{Determination of Cell Number and Proliferation}

Enterocytes were seeded at $30,000 / \mathrm{cm}^{2}$ and allowed to adhere for $48 \mathrm{~h}$, then subjected to strain or LPS $(10 \mu \mathrm{g} / \mathrm{ml})$ or their combination. Population growth was determined by staining cells with $4^{\prime}, 6$ diamidino-2-phenylindole, dihydrochloride and counting numbers of nuclei per field of view at $\times 100$ magnification for each time point with and without LPS stress. In separate wells a pulse of $1 \mathrm{mM} 5$-BrDU (Sigma-Aldrich, St Louis, MO) for $1 \mathrm{~h}$ was used before each timed end point, and BrdU was visualized using anti-BrdU antibody (Millipore, Billerica, MA) and alexafluor 488 secondary antibody (Invitrogen, Carlsbad, CA), according to manufacturers' instructions.

\section{Data Analysis}

Data were analyzed using GraphPad Prism, Version 5.0 (GraphPad Software, La Jolla, CA). Statistical comparisons were made using either $t$-tests or two way ANOVA with Bonferroni corrections where appropriate. A difference was considered significant when the $P$ value was $<0.05$.

\section{STATEMENT OF FINANCIAL SUPPORT}

This work was supported by National Institutes of Health (NIH) award 1K23DK078909-01A1 NIH.

\section{REFERENCES}

1. Halamek L, Morley C. Continuous positive airway pressure during neonatal resuscitation. Clin Perinatol 2006;33:83-98, vii.

2. Meyer M, Mildenhall L, Wong M. Outcomes for infants weighing less than 1000 grams cared for with a nasal continuous positive airway pressurebased strategy. J Paediatr Child Health 2004;40:38-41.

3. Aly H, Milner JD, Patel K, El-Mohandes AA. Does the experience with the use of nasal continuous positive airway pressure improve over time in extremely low birth weight infants? Pediatrics 2004;114:697-702.

4. De Klerk AM, De Klerk RK. Nasal continuous positive airway pressure and outcomes of preterm infants. J Paediatr Child Health 2001;37:161-7.

5. Morley CJ, Davis PG, Doyle LW, Brion LP, Hascoet JM, Carlin JB. Nasal $\mathrm{CPAP}$ or intubation at birth for very preterm infants. N Engl J Med 2008;358:700-8.

6. Jaile JC, Levin T, Wung JT, Abramson SJ, Ruzal-Shapiro C, Berdon WE. Benign gaseous distension of the bowel in premature infants treated with nasal continuous airway pressure: a study of contributing factors. AJR Am J Roentgenol 1992;158:125-7.

7. Hack M, Wright LL, Shankaran S, et al. Very-low-birth-weight outcomes of the National Institute of Child Health and Human Development 
Neonatal Network, November 1989 to October 1990. Am J Obstet Gynecol 1995;172(2 Pt 1):457-64.

8. Hsueh W, Caplan MS, Qu XW, Tan XD, De Plaen IG, Gonzalez-Crussi F. Neonatal necrotizing enterocolitis: clinical considerations and pathogenetic concepts. Pediatr Dev Pathol 2003;6:6-23.

9. Coit AK. Necrotizing enterocolitis. J Perinat Neonatal Nurs 1999;12:53-66; quiz 88-9.

10. Henry MC, Moss RL. Current issues in the management of necrotizing enterocolitis. Semin Perinatol 2004;28:221-33.

11. Lee JS, Polin RA. Treatment and prevention of necrotizing enterocolitis. Semin Neonatol 2003;8:449-59.

12. Neu J. Neonatal necrotizing enterocolitis: an update. Acta Paediatr Suppl 2005;94:100-5.

13. Stoll BJ, Hansen NI, Bell EF, et al. Neonatal outcomes of extremely preterm infants from the NICHD Neonatal Research Network. Pediatrics 2010;126:443-56.

14. Lemons J, Bauer C, Oh W, et al. Very low birth weight outcomes of the National Institute of Child Health and Human Development Research Network, January 1995 Through December 1996. Pediatrics 2001;107:E1.

15. Berman L, Moss RL. Necrotizing enterocolitis: an update. Semin Fetal Neonatal Med 2011;16:145-50.

16. Henry MC, Moss RL. Necrotizing enterocolitis. Annu Rev Med 2009;60:111-24.

17. Henry MC, Moss RL. Neonatal necrotizing enterocolitis. Semin Pediatr Surg 2008;17:98-109.

18. Miksch RM, Armbrust S, Pahnke J, Fusch C. Outcome of very low birthweight infants after introducing a new standard regime with the early use of nasal CPAP. Eur J Pediatr 2008;167:909-16.

19. Narendran V, Donovan EF, Hoath SB, Akinbi HT, Steichen JJ, Jobe AH. Early bubble CPAP and outcomes in ELBW preterm infants. J Perinatol 2003;23:195-9.
20. Aly H, Massaro AN, Patel K, El-Mohandes AA. Is it safer to intubate premature infants in the delivery room? Pediatrics 2005;115:1660-5.

21. Geary CA, Fonseca RA, Caskey MA, Malloy MH. Improved growth and decreased morbidities in $<1000 \mathrm{~g}$ neonates after early management changes. J Perinatol 2008;28:347-53.

22. Aly H, Massaro AN, Hammad TA, Narang S, Essers J. Early nasal continuous positive airway pressure and necrotizing enterocolitis in preterm infants. Pediatrics 2009;124:205-10.

23. Nanthakumar NN, Fusunyan RD, Sanderson I, Walker WA. Inflammation in the developing human intestine: A possible pathophysiologic contribution to necrotizing enterocolitis. Proc Natl Acad Sci USA 2000;97:6043-8.

24. Jilling T, Simon D, Lu J, et al. The roles of bacteria and TLR4 in rat and murine models of necrotizing enterocolitis. J Immunol 2006;177:3273-82.

25. Liu Y, Fatheree NY, Mangalat N, Rhoads JM. Human-derived probiotic Lactobacillus reuteri strains differentially reduce intestinal inflammation. Am J Physiol Gastrointest Liver Physiol 2010;299:G1087-96.

26. Meyer TA, Noguchi Y, Ogle CK, et al. Endotoxin stimulates interleukin-6 production in intestinal epithelial cells. A synergistic effect with prostaglandin E2. Arch Surg 1994;129:1290-4; discussion 1294-5.

27. Iwaki M, Ito S, Morioka M, et al. Mechanical stretch enhances IL-8 production in pulmonary microvascular endothelial cells. Biochem Biophys Res Commun 2009;389:531-6.

28. Gribar SC, Sodhi CP, Richardson WM, et al. Reciprocal expression and signaling of TLR4 and TLR9 in the pathogenesis and treatment of necrotizing enterocolitis. J Immunol 2009;182:636-46.

29. Chan KL, Wong KF, Luk JM. Role of LPS/CD14/TLR4-mediated inflammation in necrotizing enterocolitis: pathogenesis and therapeutic implications. World J Gastroenterol 2009;15:4745-52.

30. Sodhi CP, Shi XH, Richardson WM, et al. Toll-like receptor-4 inhibits enterocyte proliferation via impaired beta-catenin signaling in necrotizing enterocolitis. Gastroenterology 2010;138:185-96. 